\title{
Self-Care Practice and Associated Factors among Hypertensive Patients in Ethiopia: A Systematic Review and Meta-Analysis
}

\author{
Adam Wondmieneh $\mathbb{D},{ }^{1}$ Getnet Gedefaw $\mathbb{D}^{2},{ }^{2}$ Addisu Getie $\mathbb{D}^{1},{ }^{1}$ and Asmamaw Demis $\mathbb{D}^{1}$ \\ ${ }^{1}$ Department of Nursing, College of Health Sciences, Woldia University, P.O. Box 400, Woldia, Ethiopia \\ ${ }^{2}$ Department of Midwifery, College of Health Sciences, Woldia University, P.O. Box 400, Woldia, Ethiopia \\ Correspondence should be addressed to Adam Wondmieneh; wondmienehadam@gmail.com
}

Received 13 January 2021; Accepted 31 March 2021; Published 9 April 2021

Academic Editor: Kai Hu

Copyright (c) 2021 Adam Wondmieneh et al. This is an open access article distributed under the Creative Commons Attribution License, which permits unrestricted use, distribution, and reproduction in any medium, provided the original work is properly cited.

\begin{abstract}
Background. Hypertension is one of the leading causes of morbidity and mortality in developing countries including Ethiopia. Selfcare practice has been provided as one of the most important preventive mechanisms of hypertension and is considered as a basic treatment for hypertension. There is no national-level study that assesses hypertensive self-care practice in Ethiopia. Therefore, this study aimed to assess the pooled level of hypertensive self-care practices and associated factors in Ethiopia. Methods. This study was carried out using published and unpublished articles accessed from databases: PubMed/MEDLINE, HENARI, Google Scholar, Web of Science, Scopus, African Journals, and university repositories. Data were extracted using a standard data extraction format. Data analysis was carried out using STATA version 11. Heterogeneity across the included studies was assessed using Cochrane's Q statistics and $I^{2}$ test with its corresponding $p$ values. Publication bias was determined using Egger's test and presented with a funnel plot. The pooled level of hypertensive self-care practice was estimated using a random-effects meta-analysis model. Results. This systematic review included 17 cross-sectional studies with 5,248 study participants. The overall pooled level of self-care practice among hypertensive patients in Ethiopia was $41.55 \%$ (95\% CI 33.06, 50.05). Participant formal education (AOR $=2.82 ; 95 \%$ CI 2.18, $3.64)$ and good knowledge of hypertension $(\mathrm{AOR}=4.04 ; 95 \% \mathrm{CI} 2.19,7.44)$ were significantly associated with self-care practice among hypertensive patients in Ethiopia. Conclusion. In this study, more than half of hypertensive patients had poor hypertensive self-care practice in Ethiopia. Participant's formal education and good knowledge of hypertension were significantly associated with self-care practice among people living with hypertension in Ethiopia. Therefore, based on the evidence of this study, we recommended that programmers and policymakers should enhance the awareness of hypertensive patients on self-care practice domains and strengthen local programs working on noncommunicable diseases.
\end{abstract}

\section{Background}

Hypertension is one of the leading causes of morbidity and mortality worldwide. In 2010, a total of 1.39 billion adult population worldwide had hypertension and 1.04 billion hypertensive people were from middle- and low-income countries [1]. The prevalence of hypertension among the adult African population aged 25 and above is the highest among other regions of the world (46\%) [2]. In Ethiopia, the prevalence of hypertension was $19.6 \%$ [3]. In middle- and low-income countries including Ethiopia, the prevalence of hypertension significantly rises due to unhealthy dietary practices, the rapid expansion of urbanization, and the sedentary lifestyle of people [4-6]. People who are living in middle- and low-income countries are highly suffering from hypertension because of inadequate access to health services; low socioeconomic status of people; and low level of awareness, diagnosis, and treatment of hypertension [2, 7].

A systematic review and meta-analysis conducted in Ethiopia showed that $48 \%$ of hypertensive patients do not have their blood pressure controlled below the level of 140/ $90 \mathrm{mmHg}$ [8], which indicates that hypertension is poorly managed in Ethiopia. Uncontrolled hypertension is associated with an increased risk of cardiovascular diseases, renal diseases, eye disease, and cerebrovascular complications [9, 10]. Globally, hypertension accounts for 9.4 million 
deaths each year. Moreover, hypertension is responsible for $45 \%$ of deaths from total ischemic heart disease mortality and $51 \%$ of deaths from total cerebrovascular disease mortality [2].

Several modifiable factors are associated with a high prevalence of hypertension. These factors are excessive alcohol consumption, smoking a cigarette, overweight and obesity, inadequate access to health services, consumption of high sodium diets, and nonadherence to antihypertensive medications $[2,11]$. Evidence showed that excessive alcohol consumption was considerably associated with elevation of blood pressure $[12,13]$. Therefore, reducing alcohol consumption favors blood pressure reduction [14]. Tobacco use increases the risk of cardiovascular complications among patients with hypertension. Additionally, hypertensive patients who smoke cigarettes are at high risk of developing a severe form of hypertension including malignant hypertension [15]. Globally, cigarette smoking contributes to six million annual deaths [11]. Therefore, cessation of smoking plays a significant role to prevent the complications of hypertension.

Performing regular physical activity for 30 minutes on 5-7 days per week may be helpful for the prevention as well as the treatment of hypertension [16]. Evidence suggested that a reduction of salt intake causes a significant reduction in blood pressure [17]. In overweight and obese hypertensive patients, weight loss is associated with the reduction of blood pressure [18]. Adherence to antihypertensive medications is crucial to reduce the life-threatening complications of hypertension. However, only $54.8 \%$ of hypertensive patients adhere to antihypertensive medications [19]. Nonadherence to antihypertensive medications was significantly associated with an increased risk of stroke and cardiovascular diseases $[20,21]$.

Self-care practice has been provided as one of the most important preventive mechanisms of hypertension and is considered as a basic treatment for hypertension [16]. Self-care practices are also recommended as adjuvant therapy for those hypertensive patients who take antihypertensive medications to enhance the antihypertensive effect $[22,23]$. Hypertensive self-care practices mainly focused on weight reduction, cessation of smoking, moderation of alcohol consumption, performing regular exercise, salt reduction, and adherence to antihypertensive medications $[16,24]$. The life-threatening complications of hypertension can be reduced by improving the self-care behaviors of hypertensive patients [16]. Identification of risk factors that influence self-care practice among hypertensive patients is essential to decrease the morbidity and mortality of hypertension. Hypertensive self-care practices can be affected by several factors such as gender, age, body mass index (BMI), educational status, monthly income, knowledge on hypertension, and presence of comorbidity $[25,26]$.

In Ethiopia, several single studies reported the level of hypertensive self-care practice in different regions, which ranges from $20.3 \%$ [27] to $68.92 \%$ [28]. However, there is no national-level study that estimates the overall level of self-care practice among hypertensive patients. Estimating the overall hypertensive self-care practice is very crucial for policymakers and planners to evaluate the implementation of hypertension treatment and prevention programs and to update the current self-care practice. Therefore, this study aimed to estimate the pooled level of self-care practice and associated factors among hypertensive patients in Ethiopia.

\section{Materials and Methods}

2.1. Databases and Search Strategy. Studies reporting the level of self-care practice and/or a variety of factors associated with hypertension self-care practice in Ethiopia were systematically searched from electronic databases: PubMed/ MEDLINE, HENARI, Google Scholar, Web of Science, Scopus, African Journals, and university repositories. Additionally, the reference list of established articles was searched to identify additional literature. Studies reporting the level of self-care practice and/or recommended lifestyle among hypertensive patients in Ethiopia were included in the final meta-analysis. Searching was conducted using the following keywords: prevalence, adherence, hypertensive self-care, salt reduction, physical activity, cigarette smoking, alcohol intake, weight reduction, and medication adherence. The keywords were combined using "AND" and "OR" Boolean operators. The searching date was from November 01, 2020, to December 20, 2020, and articles accessed until December 25, 2020, were included in this systematic review and meta-analysis. The preferred reporting item for the systematic review and meta-analysis (PRISMA) guideline was used to report this systematic review and meta-analysis [29].

\subsection{Eligibility Criteria}

2.2.1. Inclusion Criteria. Published and unpublished studies conducted only in Ethiopia and satisfying the following inclusion criteria were included in this systematic review and meta-analysis.

Study design: observational studies (cross-sectional, cohort, and case-control)

Language: only studies reported in the English language

Study participants: studies were conducted among all hypertensive patients

Publication year: articles published before December 25,2020 , were included in this study

2.2.2. Exclusion Criteria. Articles that were inaccessible full text after two contacts with the primary author by e-mail were excluded. Additionally, interventional studies, systematic reviews, case reports, narrative reviews, and policy statements were excluded from this study.

2.2.3. Outcome Measurement. In this study, hypertensive self-care practice were measured using six domains: regular physical activity, salt reduction, cessation of smoking, moderation of alcohol consumption, and weight reduction [16]. 
Regular physical activity: participants performing regular physical activity $\geq 30$ minutes per day, at least 4 days per week

Salt reduction: reduce salt when preparing foods and avoid or limit the consumption of salty foods

Smoking cessation: hypertensive patients who did not smoke any pack of cigarettes

Moderation of alcohol consumption: daily alcohol consumption does not exceed 2 standard drinks per day for men and 1 standard drink per day for women (1 standard drink $=10$ gram alcohol)

Weight management: manage weight through dietary practice as well as performing exercise to lose weight

Medication adherence: hypertensive patients took medication at the recommended dosage and time

2.3. Quality Assessment. In this study, the quality of the included studies was assessed using the Newcastle-Ottawa Quality Assessment Scale for the cross-sectional study [30]. The scale has three components. The first component mainly assessed the methodological quality of each study and graded from five points. The second component graded from two points and mainly focused on the comparability of studies. The third component of the tool focused on the outcomes and statistical analysis of each article and graded from three points. Two investigators (AW and GG) assessed the quality of each study and studies with a scale of six and above six out of 10 scales were determined as high quality.

2.4. Data Extraction. The data were extracted by three independent investigators (AW, AD, and GG) using a Microsoft Excel spreadsheet. The disagreement between the investigators was resolved by the fourth author (AG) through discussion. The data extraction format included information on author name, year of publication, the region of study, study design, sample size, response rate, self-care practice, mean age, sex, residence, predictors, and each domain of hypertensive self-care practice.

2.5. Data Processing and Analysis. The pertinent data were extracted and further cleaned using a standard data extraction format, prepared in Microsoft Excel sheet, and data were exported into STATA version 11.0 (Stata Corporation, College Station, Texas) for further analysis. The data were analyzed using Der Simonian and Laird's random effect metaanalysis model at 95\% CI. Heterogeneity between the included studies was assessed using Cochrane Q statistics and $I^{2}$ percentage with its corresponding $p$-values $[31,32]$. In this study, the statistical value showed that there was high heterogeneity detected across the included studies $\left(I^{2}=97.4 \%\right.$, $p \leq 0.001)$. Therefore, a random-effects meta-analysis model was used to estimate the pooled level of hypertensive self-care practice in Ethiopia. Subgroup analysis was computed based on regions of the study, sample size, and year of publication to assess the possible source of heterogeneity detected in the pooled level of hypertensive self-care practice. Finally, the presence of publication bias was assessed using Egger's test [33] and presented graphically with funnel plots. A statistical test with a $p$-value $<0.05$ was considered statistically significant.

\section{Results}

3.1. Selection and Identification of Studies. We collected 824 articles from the different databases of PubMed/MEDLINE, HENARI, Google Scholar, Web of Science, Scopus, African Journals, and university repositories and reference lists of established articles. From these studies, 384 duplicated records were removed. Among the remaining 440 studies, 392 irrelevant studies were excluded after assessing and screening titles and abstracts. Then, 48 full-text articles were assessed for eligibility and 31 articles were further excluded for being not in line with the inclusion criteria of our study. Finally, 17 studies fulfilled the inclusion criteria and were included in this systematic review and meta-analysis (Figure 1).

3.2. Characteristics of Included Studies. In this systematic review and meta-analysis, we included 17 cross-sectional studies with 5,248 study participants. All studies were published between 2016 and 2020 in peer-reviewed journals. The minimum and maximum sample size of the included studies were 130 [34] and 422 [35], respectively. The response rate was $98.41 \%$. Half of the participants were male (50.4\%) and the mean age of the participants was 54.55 years old. The majority of the participants are living in urban areas, $69.5 \%$. The included studies were reported from five regions and one city administration of the country. Four studies were reported from the Oromia regional state $[28,34,36,37]$. Three studies were from Amhara regional state [38-40], three studies from Harari regional state $[35,41,42]$, three studies from Addis Ababa city administration [43-45], two studies from Tigray regional state $[27,46]$, and two studies from South Nations Nationalities and Peoples Regional State (SNNPR) [47, 48]. Four regions and one city administration, namely, the Afar region, Benishangul Gumuz region, Somali region, Gambella region, and Dire Dawa city administration, were not included in this study due to a lack of studies (Table 1).

3.3. Self-Care Practice among Hypertensive Patients in Ethiopia. From the total 17 included studies, 14 studies reported the level of hypertensive self-care practice in Ethiopia. The results of this meta-analysis showed that the overall estimated pooled level of self-care practice among hypertensive patients in Ethiopia was 41.55\% (95\% CI 33.06, 50.05) (Figure 2). The highest level of hypertensive self-care practice was reported from a study conducted in Nekemte (68.92\%), Oromia region, 2019 [28]. In contrast, the lowest level of hypertensive self-care practice was reported from a study done in Ayder Comprehensive Specialized Hospital (20.3\%), Tigray regional state, 2019 [27]. 


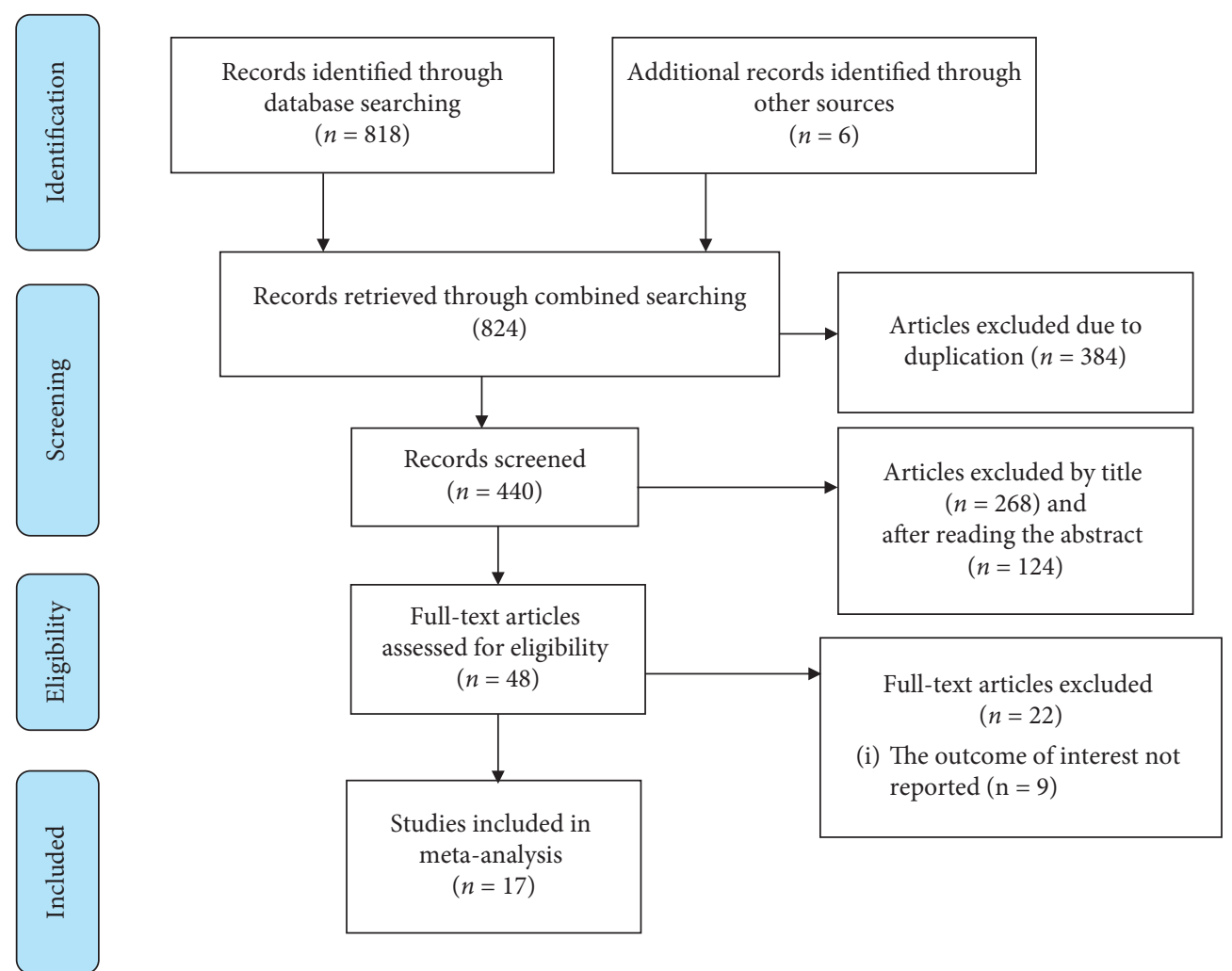

FIGURE 1: Flow chart of study selection for systematic review and meta-analysis to estimate the level of self-care practice among hypertensive patients in Ethiopia.

3.4. Subgroup Analysis. We conducted a subgroup analysis based on regions of the country where studies were conducted to investigate the possible source of heterogeneity. The results of subgroup analysis showed that the highest level of self-care practice among hypertensive patients was observed in studies conducted in Oromia regional state, 56.77 (95\% CI 33.03, 80.58) (Table 2).

\subsection{Adherence to Recommended Lifestyle Modifications among} Hypertensive Patients in Ethiopia. In the current meta-analysis, we determined the level of hypertensive self-care practice using six lifestyle recommendations: physical activity, salt reduction, cessation of smoking, moderation of alcohol intake, weight reduction, and medication adherence. Accordingly, thirteen studies included information on the physical activity of hypertensive patients. In this meta-analysis, the pooled level of performing regular physical activity was 46.68 $(35.03,58.68)$. Similarly, thirteen studies reported cessation of cigarette smoking. The pooled level of cessation of smoking among hypertensive patients was 88.09 (83.99, 92.19) (Table 3).

3.6. Heterogeneity and Publication Bias. In this study, there was high heterogeneity across the included studies according to the Cochrane $\mathrm{Q}$ test and $I^{2}$ test statistics $\left(I^{2}=97.4 \%\right.$, $p \leq 0.001$ ) [49]. Therefore, a random-effects meta-analysis model was conducted to estimate the pooled level of self-care practice among hypertensive patients. The presence of publication bias was assessed using Egger's regression test and Begg's rank correlation test. The result of Egger's weight regression $(p=0.216)$ and Begg's rank correlation test $(p=0.189)$ suggested that there was no publication bias. The funnel plot of hypertension self-care practice in Ethiopia was symmetrical, suggesting no publication bias (Figure 3 ).

3.7. Factors Associated with Hypertension Self-Care Practice in Ethiopia. A total of nine cross-sectional studies have been included to determine factors significantly associated with hypertensive self-care practice in Ethiopia. The current study identified two predictors, namely, knowledge about hypertension and the educational status of hypertensive patients. The analysis of nine cross-sectional studies $[27,35,37,39,41,43,44,47,48]$ showed that the educational status of the hypertensive patients was significantly associated with hypertensive self-care practice. Hypertensive patients who attended formal education were approximately 3 times more likely to adhere to hypertensive self-care practice than those who did not attend formal education $(\mathrm{AOR}=2.82 ; 95 \% \mathrm{CI} 2.18,3.64)$ (Figure 4). The association between hypertensive patient's good knowledge and hypertensive self-care practice was assessed by four studies $[27,41,45,48]$. The results revealed that hypertensive patients who had good knowledge of hypertension were 4 times more likely to practice self-care than those patients who had poor knowledge of hypertension $(\mathrm{AOR}=4.04 ; 95 \%$ CI 2.19, 7.44) (Figure 5). 


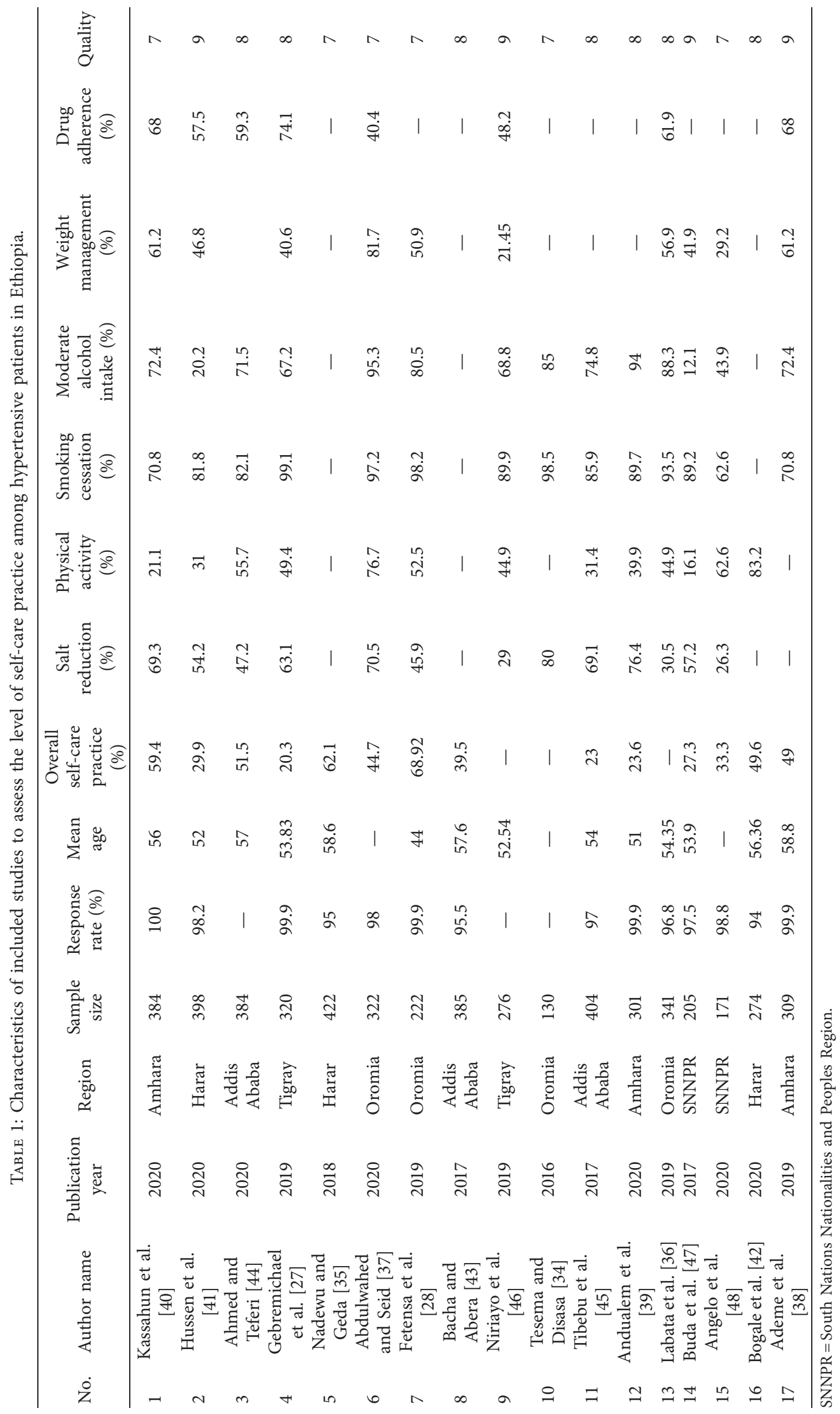




\begin{tabular}{|c|c|c|c|}
\hline \multicolumn{2}{|l|}{ Author } & \multirow{2}{*}{$\begin{array}{c}\mathrm{ES}(95 \% \mathrm{CI}) \\
59.40(54.49,64.31)\end{array}$} & \multirow{2}{*}{$\begin{array}{c}\begin{array}{c}\text { Weight } \\
(\%)\end{array} \\
7.17\end{array}$} \\
\hline Kassahun et al. (2020) & $\rightarrow-$ & & \\
\hline Hussen et al. (2020) & $\rightarrow \quad 1$ & $29.90(25.40,34.40)$ & 7.20 \\
\hline Ahmed et al. (2020) & $1 \rightarrow$ & $51.50(46.50,56.50)$ & 7.16 \\
\hline Gebremichael et al. (2019) & $\rightarrow$ & $20.30(15.89,24.71)$ & 7.20 \\
\hline Nadewu et al. (2018) & $\rightarrow$ & $62.10(57.47,66.73)$ & 7.19 \\
\hline Abdulwahed et al. (2020) & & $44.70(39.27,50.13)$ & 7.13 \\
\hline Fetensa G et al. (2019) & $\rightarrow$ & $68.92(62.83,75.01)$ & 7.08 \\
\hline Bacha et al. (2017) & & $39.50(34.62,44.38)$ & 7.17 \\
\hline Tibebu et al. (2017) & $\rightarrow-$ & $23.00(18.90,27.10)$ & 7.22 \\
\hline Andualem et al. (2020) & $\rightarrow-$ & $23.60(18.80,28.40)$ & 7.18 \\
\hline Buda et al. (2017) & $\rightarrow-$ & $27.30(21.20,33.40)$ & 7.08 \\
\hline Angelo et al. (2020) & $\rightarrow-1$ & $33.30(26.24,40.36)$ & 6.99 \\
\hline Bogale et al. (2020) & $\rightarrow$ & $49.60(43.68,55.52)$ & 7.09 \\
\hline Ademe et al. (2019) & & $49.00(43.43,54.57)$ & 7.12 \\
\hline Overall $\left(I^{2}=97.4 \%, p \leq 0.001\right)$ & & $41.55(33.06,50.05)$ & 100.00 \\
\hline Note: weights are from random effects analysis & $\begin{array}{l}1 \\
1\end{array}$ & & \\
\hline
\end{tabular}

FIGURE 2: Forest plot of included studies that assess the level of self-care practice among hypertensive patients in Ethiopia.

TABLE 2: Subgroup analysis of the estimated pooled level of self-care practice among hypertensive patients in Ethiopia.

\begin{tabular}{lccccc}
\hline Variable & Subgroup & Included studies & Sample size & Estimate of overall hypertensive self-care & $I^{2}, p$ value \\
\hline \multirow{7}{*}{ Region } & Amhara & 3 & 994 & $43.99(22.22,65.75)$ & $98.2 \%, \leq 0.001$ \\
& Harar & 3 & 1094 & $47.19(27.20,67.17)$ & $97.9 \%, \leq 0.001$ \\
& Addis Ababa & 3 & 1173 & $37.95(21.25,54.65)$ & $97.4 \%, \leq 0.001$ \\
& SNNPR & 2 & 376 & $30.02(24.17,35.88)$ & $37 \%, 0.208$ \\
\multirow{2}{*}{ Total } & Oromia & 2 & 544 & $56.77(33.03,80.58)$ & $97 \%, \leq 0.001$ \\
\hline
\end{tabular}

SNNPR: South Nations Nationalities and Peoples Region.

TABle 3: Pooled levels of adherence to the recommended self-care practice among hypertensive patients in Ethiopia.

\begin{tabular}{lcccr}
\hline Recommended lifestyle & Included studies & Sample size & Pooled level of good practice at 95\% CI & $I^{2}, p$-value \\
\hline Physical activity & 13 & 4002 & $46.68(35.03,58.68)$ & $98.6 \%, \leq 0.001$ \\
Salt reduction & 13 & 3852 & $55.03(45.58,65.03)$ & $97.7 \%, \leq 0.001$ \\
Cessation of smoking & 13 & 3852 & $88.09(83.99,92.19)$ & $97.1 \%, \leq 0.001$ \\
Moderation of alcohol intake & 13 & 3852 & $67.26(52.26,82.26)$ & $99.4 \%, \leq 0.001$ \\
Adherence to medications & 7 & 2232 & $58.55(50.26,66.84)$ & $94.7 \%, \leq 0.001$ \\
Weight reduction & 9 & 2446 & $47.90(34.83,60.97)$ & $98.1 \%, \leq 0.001$ \\
\hline
\end{tabular}

\section{Discussion}

Hypertensive self-care practice is very important for the prevention and treatment of hypertension [16]. As far as the investigator's knowledge, this study is the first of its kind to estimate the pooled level of hypertensive self-care practice in Ethiopia. Estimating the overall level of self-care practice among hypertensive patients is very crucial for hypertension treatment and prevention of complications related to hypertension. Adherence to the recommended hypertensive self-care practice requires continuous motivation and education from different stakeholders [14].

The findings of this study showed that more than half of hypertensive patients had poor hypertensive self-care practice in Ethiopia $41.55 \%$ (95\% CI 33.06, 50.05). The current finding is in line with a study done in West Bagel, India (37.1\%). However, this finding was lower than studies conducted in Ghana (72\%) [50], Tertiary Level Hospital of Kathmandu, Nepal (57.1\%) [51], and South India (60.6\%) [52], and much higher than a study done in Nigeria (16.4\%) [53]. The possible explanation for this 


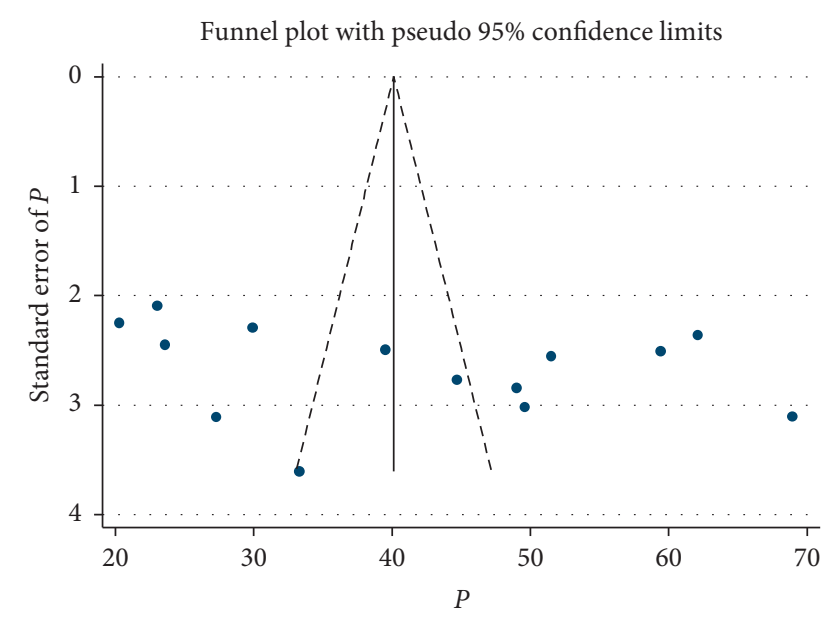

Figure 3: Funnel plot to assess the level of self-care practice among hypertensive patients in Ethiopia.

variation might be due to a difference in socioeconomic status, cultural practices, educational status, access to health care facilities, and lifestyle of the study participants. Additionally, there was methodological variation and differences in the components of the hypertension self-care practice assessment tool.

In the current meta-analysis, more than half of the study participants have not performed regular physical activity 46.68 (95\% CI: 35.03, 58.68). This finding is supported by a study done in Nepal (44.8\%) [51] and America (52.2\%) [54]. However, this finding is lower than a study done in Saudi Arabia (20.1\%) [25]. This variation might be due to a lack of organized set up around the living areas for exercise and there are cultural differences. Aerobic exercise is an effective treatment for lowering blood pressure in hypertensive patients [55]. Therefore, performing a regular aerobic exercise for 30 minutes on 5-7 days per week may be important for both the prevention and treatment of hypertension [16].

In this meta-analysis, more than half of the hypertensive patients practiced the recommended salt reduction of 55.03\% (95\% CI: 45.58, 65.03). Evidence from the Cochrane library showed that dietary sodium intake reduction from 11.59 grams per day to 3.89 grams per day reduces blood pressure by $3 \%$ in hypertensive patients [56]. Reduction of the daily intake of salt by 5 grams decreases the risk of cardiovascular and stroke mortality by $17 \%$ and $23 \%$, respectively [57]. The American College of Cardiology 2017 Guideline for the treatment and prevention of hypertension recommended that the daily intake of salt should not exceed 1.5 grams [58]. Therefore, the reduction of salt during food preparation and avoidance or limitation of consumption of salty foods may be beneficial for both in the reduction of mortality and morbidity in hypertensive patients.

In the current study, 47.9\% (95\% CI, 34.83 60.97) of hypertensive patients have practiced the recommended weight reduction. A similar finding was observed in a study conducted in Nepal (48.6\%) [51]. A study conducted among African American hypertensive patients showed that only $30.1 \%$ of participants practiced weight reduction [54]. The current finding is much higher than a study conducted in India (11.4\%)
[59]. This variation could be explained by cultural differences. Additionally, there is methodological variation, in which this study analyzed the level of hypertensive self-care practices with a large sample size $(5,248)$, while the above-mentioned studies were conducted at a specific place with a small sample size.

The results of this study showed that the majority of the study participants $88.09 \%$ (95\% CI: 83.99, 92.19) were not smoking a cigarette. Similar findings were reported from a study done in Israel (87\%) [60] and Nepal (84.9\% [51]. The results of this meta-analysis showed that $11.91 \%$ of respondents still smoke a cigarette. A 15-year population-based cohort study conducted in Indonesia showed that cigarette smoking was significantly correlated with high blood pressure [61]. Therefore, cessation of smoking is crucial for the reduction of cardiovascular and cerebrovascular complications of hypertension [15].

In this meta-analysis, $67.26 \%$ (95\% CI: 52.26, 82.26) of hypertensive patients were adherent to moderate alcohol intake. The result of this study is supported by a study conducted in the USA among African American study participants (65\%) [54]. A cross-sectional study done in Nepal showed that $85.8 \%$ of study participants avoid alcohol consumption [51]. The American College of Cardiology recommended that daily alcohol intake should not exceed 2 drinks per day for men and one drink per day for women [58]. Reducing alcohol consumption lowers the blood pressure in people who drink more than two drinks per day [62]. Therefore, reducing alcohol intake is important to decrease the burden of hypertension.

In the current study, more than half of hypertensive patients $58.55 \%$ (95\% CI: 50.26, 66.84) were adherent to antihypertensive medications. This finding is in line with a study conducted in India (54\%) [59] and in America, 2011 (58.6\%) [54]. However, this finding is lower than a study reported from America in 2015 (69\%) [63] and Korea $(81.7 \%)$ [64]. In contrast, this finding is higher than a study reported from Egypt (46.12\%) [65] and Palestine (45.8\%) [66]. The discrepancy might be due to differences in the socioeconomic status of participants. Moreover, there is a methodological variation between this study and the abovementioned studies. Therefore, improving adherence to antihypertensive medications is crucial to decrease complications of uncontrolled hypertension.

In this systematic review and meta-analysis, knowledge on hypertension and the educational status of hypertensive patients were significant predictors of hypertensive self-care practice in Ethiopia. Hypertensive patients who attended formal education were approximately 3 times more likely to adhere to hypertensive self-care practice than those who did not attend formal education. This finding is supported by studies done in the Ashanti region of Ghana [50] and West Bengal, India [67]. A study conducted in Nepal showed that less-educated hypertensive patients were not adhering to hypertensive self-care practices like educated hypertensive patients [51]. This may be due to the fact that illiterate and less educated hypertensive individuals may not understand the benefits of self-care practices and the complications of poorly controlled and uncontrolled hypertension. Moreover, educated people can read and easily understand the 


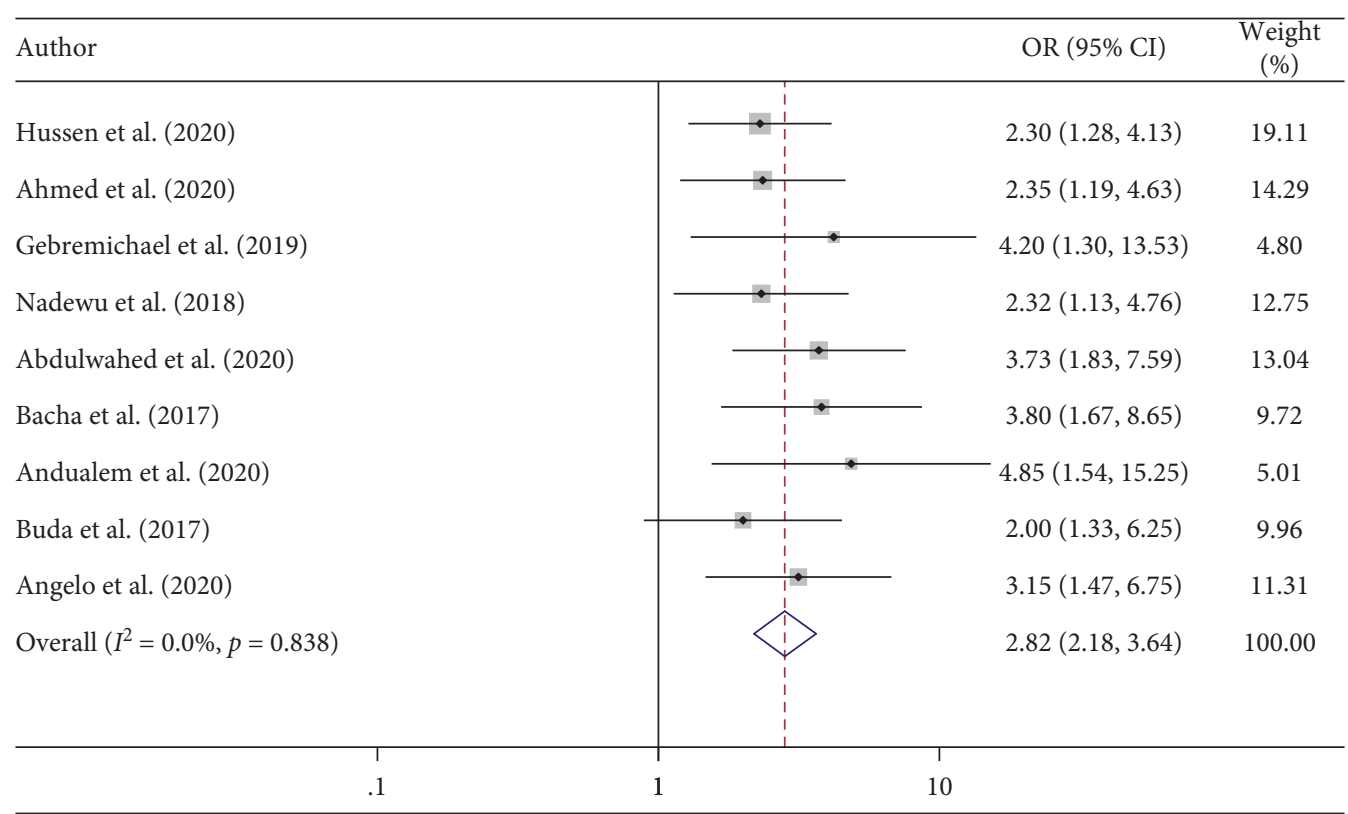

FIgURE 4: The pooled odds ratio of the association between educational status and self-care practice among hypertensive patients in Ethiopia.

\begin{tabular}{|c|c|c|}
\hline Author & OR $(95 \%$ CI $)$ & $\begin{array}{c}\text { Weight } \\
(\%)\end{array}$ \\
\hline Hussen et al. (2020) & $4.70(2.51,8.82)$ & 29.97 \\
\hline Gebremichael et al. (2019) & $6.20(2.91,13.23)$ & 26.31 \\
\hline Tibebu et al. (2017) & $13.27(4.12,42.73)$ & 17.04 \\
\hline Angelo et al. (2020) & $2.25(1.07,4.74)$ & 26.68 \\
\hline Overall $\left(I^{2}=58.9 \%, p=0.063\right)$ & $4.96(2.67,9.21)$ & 100.00 \\
\hline Note: weights are from random effects analysis & & \\
\hline
\end{tabular}

Figure 5: The pooled odds ratio of the association between hypertensive patient's knowledge and self-care practice among hypertensive patients in Ethiopia.

recommended hypertensive treatment guidelines, which in turn improve self-care practice of hypertensive patients $[68,69]$. Therefore, enhancing adult education has a significant impact to increase hypertensive self-care practices.

Finally, the results of this meta-analysis showed that hypertensive patients who had good knowledge about hypertension were 4 times more likely to practice self-care than those patients who had poor knowledge about hypertension. Similar findings were reported to form studies done in Israel [60]. Good knowledge about hypertension promotes patients to give more emphasis on hypertensive self-care practice. A randomized controlled trial conducted among hypertensive women showed that improving the knowledge of hypertensive patients through a participatory method of education could help hypertensive patients to practice better self-care and reduce disease complications [70].

\section{Limitations of the study}

The limitation of this meta-analysis in all regions of the country was not represented due to a lack of studies. Besides, the included studies reported the government health facility population and so this study does not consider hypertension patients who have follow-up in 
private clinics and home-dwelling people. Finally, all included studies have a cross-sectional study design, which shares the limitations of the cross-sectional study design.

\section{Conclusion}

In this study, more than half of the hypertensive patients had poor hypertensive self-care practice in Ethiopia. Participant's educational status and knowledge on hypertension were significantly associated with self-care practice among people living with hypertension in Ethiopia. Therefore, based on the evidence of this study, we recommend that programmers and policymakers should enhance the awareness of hypertensive patients on self-care practice domains and strengthen local programs working on noncommunicable diseases. Moreover, national populationbased studies must be conducted to assess the risk factors that contribute to poor self-care practice among hypertensive patients in Ethiopia.

\section{Abbreviations}

AOR: $\quad$ Adjusted odds ratio

BMI: $\quad$ Body mass index

CI: $\quad$ Confidence interval

PRISMA: Preferred reporting item for systematic review and meta-analysis

SNNPR: South Nation Nationalities and Peoples Region.

\section{Data Availability}

All related data have been presented within the manuscript. The datasets supporting the conclusions of this article are available from the authors on request.

\section{Conflicts of Interest}

The authors declare that they have no conflicts of interest.

\section{Authors' Contributions}

AW and AD designed the study. AW and AD designed and run the literature search. All authors (AW, AG, GG, and AD) acquired data, screened records, extracted data, and assessed the quality of each study. AD and AW did the statistical analysis and wrote the report. All authors provided critical conceptual input, analyzed and interpreted the data, and critically revised the report. Finally, all authors read and approved the final manuscript before submission to this journal.

\section{References}

[1] K. T. Mills, A. Stefanescu, and J. He, "The global epidemiology of hypertension," Nature Reviews Nephrology, vol. 16, no. 4, pp. 223-237, 2020.

[2] World Health Organization, A Global Brief on Hypertension: Silent Killer, Global Public Health Crisis: World Health Day 2013, World Health Organization, Geneva, Switzerland, 2013.
[3] K. T. Kibret and Y. M. Mesfin, "Prevalence of hypertension in Ethiopia: a systematic meta-analysis," Public Health Reviews, vol. 36, no. 1, pp. 1-12, 2015.

[4] K. Juma, P. A. Juma, C. Shumba et al., "Non-communicable diseases and urbanization in African cities: a narrative review," in Non-communicable Diseases and Urbanization-A Global PerspectiveIntechOpen, London, UK, 2019.

[5] V. Mwenda, M. Mwangi, L. Nyanjau et al., "Dietary risk factors for non-communicable diseases in Kenya: findings of the STEPS survey, 2015," BMC Public Health, vol. 18, no. 3, p. $1218,2018$.

[6] B. Twinamasiko, E. Lukenge, S. Nabawanga et al., "Sedentary lifestyle and hypertension in a periurban area of Mbarara, South western Uganda: a population based cross sectional survey," International Journal of Hypertension, vol. 2018, Article ID 8253948, 8 pages, 2018.

[7] J. Kayima, R. K. Wanyenze, A. Katamba et al., "Hypertension awareness, treatment and control in Africa: a systematic review," BMC Cardiovascular Disorders, vol. 13, no. 1, p. 54, 2013.

[8] F. Amare, B. Hagos, M. Sisay, and B. Molla, "Uncontrolled hypertension in Ethiopia: a systematic review and metaanalysis of institution-based observational studies," $B M C$ Cardiovascular Disorders, vol. 20, no. 1, pp. 1-9, 2020.

[9] D. Zhou, B. Xi, M. Zhao et al., "Uncontrolled hypertension increases risk of all-cause and cardiovascular disease mortality in US adults: the NHANES III linked mortality study," Scientific Reports, vol. 8, no. 1, pp. 1-7, 2018.

[10] G. DanaeiM. Ezzati et al., "Cardiovascular disease, chronic kidney disease, and diabetes mortality burden of cardiometabolic risk factors from 1980 to 2010: a comparative risk assessment," Lancet Diabetes \& Endocrinology, vol. 2, no. 8, pp. 634-647, 2014.

[11] World Health Organization, Global Status Report on Noncommunicable Diseases 2014, World Health Organization, Geneva, Switzerland, 2014.

[12] N. M. T. Santana, J. G. Mill, G. Velasquez-Melendez et al., "Consumption of alcohol and blood pressure: results of the ELSA-Brasil study,” PLoS One, vol. 13, no. 1, Article ID e0190239, 2018.

[13] P. M. Miller, R. F. Anton, B. M. Egan, J. Basile, and S. A. Nguyen, "Excessive alcohol consumption and hypertension: clinical implications of current research," The Journal of Clinical Hypertension, vol. 7, no. 6, pp. 346-351, 2005.

[14] S. Mahmood, K. U. Shah, T. M. Khan et al., "Non-pharmacological management of hypertension: in the light of current research," Irish Journal of Medical Science, vol. 188, no. 2, pp. 437-452, 2019.

[15] A. Virdis, C. Giannarelli, M. Fritsch Neves, S. Taddei, and L. Ghiadoni, "Cigarette smoking and hypertension," Current Pharmaceutical Design, vol. 16, no. 23, pp. 2518-2525, 2010.

[16] T. Unger, C. Borghi, F. Charchar et al., "2020 international society of hypertension global hypertension practice guidelines," Hypertension, vol. 75, no. 6, pp. 1334-1357, 2020.

[17] F. J. He, J. Li, and G. A. MacGregor, "Effect of longer term modest salt reduction on blood pressure: Cochrane systematic review and meta-analysis of randomised trials," BMJ, vol. 346, p. f1325, 2013.

[18] G. A. Bray, D. H. Ryan, and D. W. Harsha, "Diet, weight loss, and cardiovascular disease prevention," Current Treatment Options in Cardiovascular Medicine, vol. 5, no. 4, pp. 259-269, 2003. 
[19] T. M. Abegaz, A Shehab, E. A. Gebreyohannes et al., "Nonadherence to antihypertensive drugs: a systematic review and meta-analysis," Medicine, vol. 96, no. 4, 2017.

[20] S. Shin, H. Song, S.-K. Oh, K. E. Choi, H. Kim, and S. Jang, "Effect of antihypertensive medication adherence on hospitalization for cardiovascular disease and mortality in hypertensive patients," Hypertension Research, vol. 36, no. 11, pp. 1000-1005, 2013.

[21] H. J. Lee, S.-I. Jang, and E.-C. Park, "Effect of adherence to antihypertensive medication on stroke incidence in patients with hypertension: a population-based retrospective cohort study," BMJ Open, vol. 7, no. 6, Article ID e014486, 2017.

[22] R. Oza and M. Garcellano, "Nonpharmacologic management of hypertension: what works?" American Family Physician, vol. 91, no. 11, pp. 772-776, 2015.

[23] J. L. Jameson, Harrison's Principles of Internal Medicine, McGraw-Hill Education, New York, NY, USA, 2018.

[24] S. O. Ike, P. N. Aniebue, and U. U. Aniebue, "Knowledge, perceptions and practices of lifestyle-modification measures among adult hypertensives in Nigeria," Transactions of the Royal Society of Tropical Medicine and Hygiene, vol. 104, no. 1, pp. 55-60, 2010.

[25] A. I. Elbur, "Level of adherence to lifestyle changes and medications among male hypertensive patients in two hospitals in Taif; Kingdom of Saudi Arabia," International Journal of Pharmacy and Pharmaceutical Sciences, vol. 7, no. 4, pp. 168-172, 2015.

[26] R. AlHadlaq, M. Swarelzahab, S. AlSaad et al., "Factors affecting self-management of hypertensive patients attending family medicine clinics in Riyadh, Saudi Arabia," Journal of Family Medicine and Primary Care, vol. 8, no. 12, p. 4003, 2019.

[27] G. B. Gebremichael, K. K. Berhe, B. G. Beyene, and K. B. Gebrekidan, "Self-care practices and associated factors among adult hypertensive patients in Ayder Comprehensive Specialized Hospital, Tigray, Ethiopia, 2018," BMC Research Notes, vol. 12, no. 1, p. 489, 2019.

[28] G. Fetensa, N. Milkiyas, M. Besho et al., "Assessment of knowledge and practice of life style modification among hypertensive patients at Nekemte specialized hospital, western Oromia, Ethiopia: a cross-sectional study design," Journal of Cardiovascular Diseases \& Diagnosis, vol. 7, no. 6, 2019.

[29] D. Moher, A. Liberati, J. Tetzlaff et al., "Preferred reporting items for systematic reviews and meta-analyses: the PRISMA statement," PLoS Medicine, vol. 6, no. 7, Article ID e1000097, 2009.

[30] Newcastle-Ottawa Quality Assessment Scale (Adapted for Cross Sectional Studies), 2020, https://journals.plos.org/ plosone/article/file?type=supplementary\&id=info:doi/10. 1371/journal.pone.0147601.s001.

[31] M. Borenstein, L. V. Hedges, J. P. T. Higgins, and H. R. Rothstein, "A basic introduction to fixed-effect and random-effects models for meta-analysis," Research Synthesis Methods, vol. 1, no. 2, pp. 97-111, 2010.

[32] G. Rücker, G. Schwarzer, J. R. Carpenter, and M. Schumacher, "Undue reliance on $I^{2}$ in assessing heterogeneity may mislead," BMC Medical Research Methodology, vol. 8, no. 1, p. 79, 2008.

[33] M. Egger, G. D. Smith, M. Schneider, and C. Minder, "Bias in meta-analysis detected by a simple, graphical test," $B M J$, vol. 315, no. 7109, pp. 629-634, 1997.

[34] S. Tesema and B. Disasa, "Knowledge, attitude and practice regarding lifestyle modification of hypertensive patients at jimma university specialized hospital, Ethiopia," Primary Healthcare: Open Access, vol. 6, no. 1, 2016.

[35] A. N. Nadewu and B. Geda, "Adherence to healthy lifestyle among hypertensive patients in Harar region, eastern Ethiopia," Primary Health Care Open Access, vol. 8, no. 4, 2018.

[36] B. G. Labata, M. B. Ahmed, G. F. Mekonen, and F. B. Daba, "Prevalence and predictors of self care practices among hypertensive patients at Jimma University Specialized Hospital, Southwest Ethiopia: cross-sectional study," BMC Research Notes, vol. 12, no. 1, p. 86, 2019.

[37] A. Abdulwahed and A. Seid, "Level of self-care practice and associated factors among hypertensive patients in Jimma university specialized hospital, south west Ethiopia," Rehabilitation Science, vol. 5, no. 2, p. 12, 2020.

[38] S. Ademe, F. Aga, and D. Gela, "Hypertension self-care practice and associated factors among patients in public health facilities of Dessie town, Ethiopia," BMC Health Services Research, vol. 19, no. 1, p. 51, 2019.

[39] A. Andualem, H. Gelaye, and Y. Damtie, "Adherence to lifestyle modifications and associated factors among adult hypertensive patients attending chronic follow-up units of dessie referral hospital, north east Ethiopia, 2020," Integrated Blood Pressure Control, vol. 13, pp. 145-156, 2020.

[40] C. W. Kassahun, A. Asasahegn, D. Hagos et al., "Knowledge on hypertension and self-care practice among adult hypertensive patients at university of Gondar comprehensive specialized hospital, Ethiopia, 2019," International Journal of Hypertension, vol. 2020, Article ID 5649165, 7 pages, 2020.

[41] F. M. Hussen, H. A. Adem, H. S. Roba et al., "Self-care practice and associated factors among hypertensive patients in public health facilities in Harar Town, Eastern Ethiopia: a cross-sectional study," SAGE Open Medicine, vol. 8, Article ID 2050312120974145, 2020.

[42] S. Bogale, K. M. Mishore, A. Tola et al., "Knowledge, attitude and practice of lifestyle modification recommended for hypertension management and the associated factors among adult hypertensive patients in Harar, Eastern Ethiopia," SAGE Open Medicine, vol. 8, Article ID 2050312120953291, 2020.

[43] D. Bacha and H. Abera, "Knowledge, attitude and self-care practice towards control of hypertension among hypertensive patients on follow-up at St. Paul's hospital, Addis Ababa," Ethiopian Journal of Health Sciences, vol. 29, no. 4, pp. 421-430, 2019.

[44] S. M. Ahmed and M. B. Teferi, "Assessment of knowledge, self-care practice, and associated factors among hypertensive patients the public hospital of Addis Ababa Ethiopia 2016 G.C." International Journal of Cardiovascular and Thoracic Surgery, vol. 6, no. 2, p. 28, 2020.

[45] A. Tibebu, D. M. Bekele, and L. Negesa, "Adherence to recommended lifestyle modifications and factors associated for hypertensive patients attending chronic follow-up units of selected public hospitals in Addis Ababa, Ethiopia," Patient Preference and Adherence, vol. 11, pp. 323-330, 2017.

[46] Y. L. Niriayo, S. Ibrahim, T. D. Kassa et al., "Practice and predictors of self-care behaviors among ambulatory patients with hypertension in Ethiopia," PLoS One, vol. 14, no. 6, Article ID e0218947, 2019.

[47] E. S. Buda, L.K. Hanfore, R. O. Fite, and A. S. Buda, "Lifestyle modification practice and associated factors among diagnosed hypertensive patients in selected hospitals, South Ethiopia," Clinical Hypertension, vol. 23, p. 26, 2017.

[48] A. T. Angelo and T. E. Geltore, "Lifestyle modification practice and associated factors among diagnosed hypertensive 
patients in Mizan Tepi University Teaching Hospital South west Ethiopia, 2019: cross-sectional study," PAMJ Clinical Medicine, vol. 2, 2020.

[49] J. P. Higgins and S. Green, Cochrane Handbook for Systematic Reviews of Interventions, John Wiley \& Sons, Hoboken, NJ, USA, 2019.

[50] Y. Obirikorang, C. Obirikorang, E. Acheampong et al., "Adherence to lifestyle modification among hypertensive clients: a descriptive cross-sectional study," OALib, vol. 5, no. 2, pp. 1-13, 2018.

[51] G. K. Satyal, L. Rai, R. Gautam et al., "Knowledge and self-care practice on hypertension among hypertensive patients in a tertiary level hospital of Kathmandu," Journal of Institute of Medicine Nepal, vol. 42, no. 2, 2020.

[52] N. Joseph, M. Chiranjeevi, S. Sen et al., "Awareness on hypertension and its self-management practices among hypertensive patients attending outreach clinics of a medical college in south India," Kathmandu University Medical Journal, vol. 55, no. 3, pp. 202-209, 2016.

[53] G. Iloh, "Adherence to lifestyle modifications among adult hypertensive Nigerians with essential hypertension in a primary care clinic of a tertiary hospital in resource-poor environment of Eastern Nigeria," British Journal of Medicine and Medical Research, vol. 4, no. 18, pp. 3478-3490, 2014.

[54] J. Warren-Findlow and R. B. Seymour, "Prevalence rates of hypertension self-care activities among African Americans," Journal of the National Medical Association, vol. 103, no. 6, pp. 503-512, 2011.

[55] L. Cao, X. Li, P. Yan et al., "The effectiveness of aerobic exercise for hypertensive population: a systematic review and meta-analysis," The Journal of Clinical Hypertension, vol. 21, no. 7, pp. 868-876, 2019.

[56] N. A. Graudal, T. Hubeck-Graudal, and G. Jurgens, "Effects of low sodium diet versus high sodium diet on blood pressure, renin, aldosterone, catecholamines, cholesterol, and triglyceride," Cochrane Database of Systematic Reviews, vol. 12, 2020.

[57] World Health Organization, Noncommunicable Disease Education Manual for Primary Health Care Professionals and Patients, World Health Organization, Geneva, Switzerland, 2017.

[58] American College of Cardiology, "Guideline for the prevention, detection, evaluation, and management of high blood pressure in adults. Evaluation, and management of high blood pressure in adults," Journal of the American College of Cardiology, vol. 23976, 2017.

[59] S. Chacko and P. Jeemon, "Role of family support and self-care practices in blood pressure control in individuals with hypertension: results from a cross-sectional study in Kollam District, Kerala," Welcome Open Research, vol. 5, 2020.

[60] A. D. Heymann, R. Gross, H. Tabenkin et al., "Factors associated with hypertensive patients' compliance with recommended lifestyle behaviors," IMAJ-Israel Medical Association Journal, vol. 13, no. 9, p. 553, 2011.

[61] H. Andriani, R. I. Kosasih, S. Putri, H.-W. Kuo et al., "Effects of changes in smoking status on blood pressure among adult males and females in Indonesia: a 15-year population-based cohort study," BMJ Open, vol. 10, no. 4, Article ID e038021, 2020.

[62] M. Roerecke, J. Kaczorowski, S. W. Tobe, G. Gmel, O. S. M. Hasan, and J. Rehm, "The effect of a reduction in alcohol consumption on blood pressure: a systematic review and meta-analysis," The Lancet Public Health, vol. 2, no. 2, pp. e108-e120, 2017.
[63] T. E. Chang, M. D. Ritchey, S. Park et al., "National rates of nonadherence to antihypertensive medications among insured adults with hypertension, 2015," Hypertension, vol. 74, no. 6, pp. 1324-1332, 2019.

[64] H. Y. Choi, I. J. Oh, J. A. Lee et al., "Factors affecting adherence to antihypertensive medication," Korean Journal of Family Medicine, vol. 39, no. 6, p. 325, 2018.

[65] A. Hussein, M. S. Awad, and H. E. M. Mahmoud, "Patient adherence to antihypertensive medications in upper Egypt: a cross-sectional study," The Egyptian Heart Journal, vol. 72, pp. 1-8, 2020.

[66] R. Al-Ramahi, "Adherence to medications and associated factors: a cross-sectional study among Palestinian hypertensive patients," Journal of Epidemiology and Global Health, vol. 5, no. 2, pp. 125-132, 2015.

[67] A. Dasgupta, S. Sembiah, B. Paul, A. Ghosh, B. Biswas, and N. Mallick, "Assessment of self-care practices among hypertensive patients: a clinic based study in rural area of Singur, West Bengal," International Journal of Community Medicine and Public Health, vol. 5, no. 1, pp. 262-267, 2017.

[68] D. M. Neminqani, E. A. El-Shereef, and M. Thubiany, "Hypertensive patients: self-care management practices in Al-Taif, KSA," International Journal of Scientific Research, vol. 12, no. 4, pp. 1705-1714, 2013.

[69] N. Akhter, Self-management Among Patient with Hypertension in Bangladesh, Prince of Songkla University, Hat Yai, Thailand, 2010.

[70] S. S. Daniali, A. A. Eslami, M. R. Maracy, J. Shahabi, and F. Mostafavi-Darani, "The impact of educational intervention on self-care behaviors in overweight hypertensive women: a randomized control trial," ARYA Atherosclerosis, vol. 13, no. 1, p. 20, 2017. 